\title{
Coupled Resonator Vertical Cavity Laser Diode
}

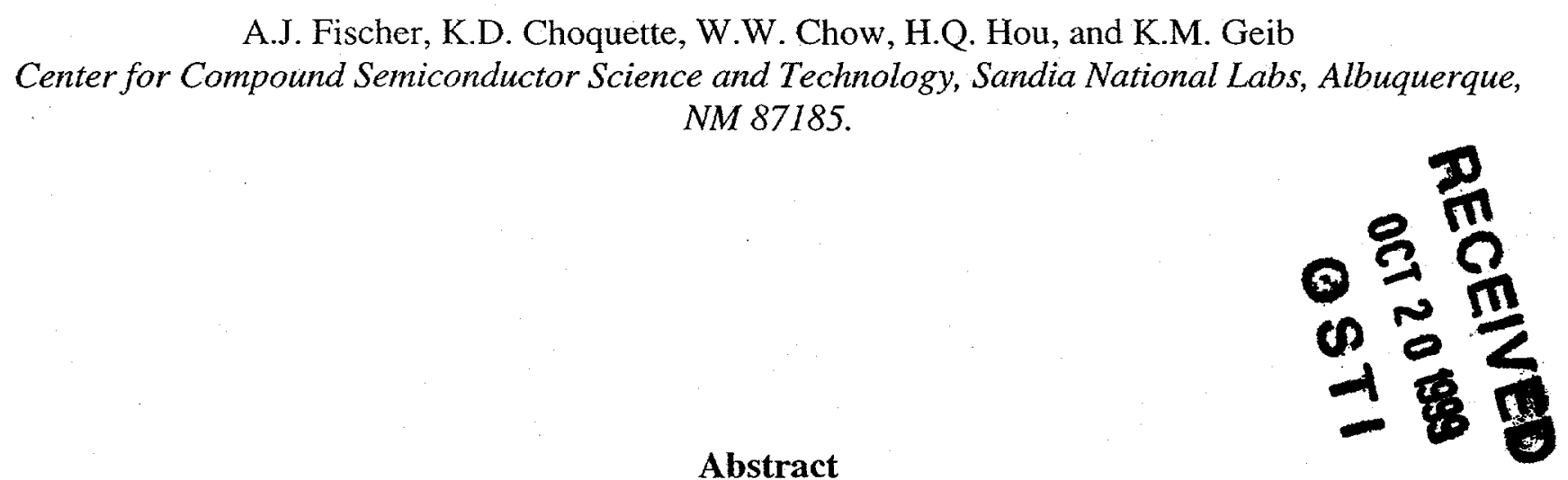

We report the operation of an electrically injected monolithic coupled resonator vertical cavity laser which consists of an active cavity containing $\operatorname{In}_{x} \mathrm{Ga}_{1-\mathrm{x}}$ As quantum wells optically coupled to a passive GaAs cavity. This device demonstrates novel modulation characteristics arising from dynamic changes in the coupling between the active and passive cavities. A composite mode theory is used to model the output modulation of the coupled resonator vertical cavity laser. It is shown that the laser intensity can be modulated by either forward or reverse biasing the passive cavity. Under forward biasing, the modulation is due to carrier induced changes in the refractive index, while for reverse bias operation the modulation is caused by field dependent cavity enhanced absorption.

Keywords: vertical cavity surface emitting lasers, VCSEL, coupled resonator, coupled cavity 
For many applications, the device performance of edge emitting semiconductor lasers can be significantly improved through the use of multiple section devices. For example, cleaved coupled cavity lasers have been shown to provide single mode operation, wavelength tuning, high speed switching, as well as the generation of short pulses via mode-locking and Q-switching [1]. th

Recently, composite resonator vertical cavity lasers have been fabricated and studied via optical Hat

pumping [24] and, more recently, under electrical injection [5,6]. Vertical cavity lasers have many (3)

advantages over edge emitters, which make them attractive for use in coupled cavity structures.

These include monolithic growth of the coupled resonator, the ability to easily modify the amount of coupling, and the ability to fabricate 2 -dimensional arrays. Coupled vertical cavity structures have demonstrated three mode coupling (two photonic and one excitonic)[2], dual wavelength emission $[3,4,6]$, and generation of picosecond pulses [4].

Previously, we demonstrated the first electrically injected coupled resonator vertical cavity laser (CRVCL) [5]. In this paper, both theoretical and experimental modulation properties of CRVCL devices are investigated in an active-passive cavity configuration. Efficient modulation of the laser emission can be achieved by either forward or reverse biasing the passive cavity. It should be noted that a vertical cavity laser with an integrated modulator has been previously reported [7], but cavity coupling was not fully exploited. The CRVCL devices discussed in this paper use a novel mechanism based on changes in cavity coupling to modulate the output intensity.

Fig. 1 shows a schematic of the CRVCL structure, which consists of a lower $1-\lambda$ thick active resonator containing three $8 \mathrm{~nm} \operatorname{In}_{x} \mathrm{Ga}_{1-x} A s$ quantum wells and a passive upper resonator composed of $1 / 2-\lambda$ thick bulk GaAs. The structure was grown by metalorganic vapor phase epitaxy (MOVPE) with a bottom p-type distributed Bragg reflector (DBR), a middle n-type DBR, and a top p-type DBR with periods of $34,11.5$ and 21 , respectively. The coupling between the two cavities can be 


\section{DISCLAIMER}

This report was prepared as an account of work sponsored by an agency of the United States Government. Neither the United States Government nor any agency thereof, nor any of their employees, make any warranty, express or implied, or assumes any legal liability or responsibility for the accuracy, completeness, or usefulness of any information, apparatus, product, or process disclosed, or represents that its use would not infringe privately owned rights. Reference herein to any specific commercial product, process, or service by trade name, trademark, manufacturer, or otherwise does not necessarily constitute or imply its endorsement, recommendation, or favoring by the United States Government or any agency thereof. The views and opinions of authors expressed herein do not necessarily state or reflect those of the United States Government or any agency thereof. 


\section{DISCLAIMER}

Portions of this document may be illegible in electronic image products. Images are produced from the best available original document. 
accurately controlled by changing the number of periods of the middle n-DBR. In the bottom active cavity we employ selective oxidation of AlGaAs to form buried oxide layers for efficient electrical and optical confinement [8]. Separate electrical contacts to each DBR provide independent current injection into the two resonators, thus producing a three terminal optoelectronic source. We show in the following that current injection into the passive cavity can be used to modulate the laser emission.

Fig. 2 shows the measured reflectance of a CRVCL structure. The inset of Fig. 2 shows the calculated cavity resonances as a function of periods in the middle DBR. The cavity resonances are determined by the transmission of the shared middle DBR. Our experimental separation of $14 \mathrm{~nm}$ for an 11.5 period DBR agrees well with the calculation. In this active-passive configuration where the bulk GaAs passive cavity does not provide useful gain, lasing is observed only from the longer wavelength resonance.

Fig. 3 (a) shows the CRVCL output intensity as a function of active current for a $20 \times 20 \mu \mathrm{m}$ oxide aperture (lower cavity) at various currents in the $58 \times 58 \mu \mathrm{m}$ passive cavity (upper cavity). The maximum laser intensity decreases with increasing forward bias current applied to the passive cavity. As carriers are injected into the passive cavity, the index of refraction of the bulk GaAs layer is depressed causing a change in the optical path length of the passive cavity. The amount of coupling between the two cavities is changed so that a smaller fraction of the lasing mode remains in the passive cavity, which leads to a reduced output intensity.

It is well known that heating in a VCSEL causes the lasing wavelength to red shift with increasing applied current until the emission is finally extinguished due to the spectral misalignment of the laser gain and the cavity resonance [10]. In order to determine if the modulation observed in Fig. 3 (a) is due to a depression of the refractive index, the fundamental lasing mode was tracked as 
a function of active cavity currents for various passive cavity currents. Fig. 4 shows the lasing wavelength for several different operating conditions along the light vs. current curve. When no current is applied to the passive cavity and the output intensity is near its maximum value (position A in Fig. 4), the wavelength of the fundamental mode is $998.7 \mathrm{~nm}$. If the active cavity current is increased to $60 \mathrm{~mA}$ (position $\mathrm{B}$ ), the nearly suppressed lasing wavelength is $1001.6 \mathrm{~nm}$, which is a change of $2.9 \mathrm{~nm}$. Alternatively, we can again start from point $A$ and inject $20 \mathrm{~mA}$ into the passive cavity (position $C$ ) producing a wavelength shift of only $1.1 \mathrm{~nm}$. The reduced spectral shift as compared to the thermally induced misalignment shows that the device is not merely driven into thermal shutdown by the heat from the passive cavity. Furthermore, since changes in the coupling between resonators may change the emission wavelength, it is difficult to separate what fraction of the modulation is due to thermal effects and what fraction is due to carrier induced changes. We expect that heat produced in the passive cavity will reduce the output emission, however, heating alone cannot explain the modulation observed in Fig. 3 (a).

The modulation mechanism can be described in terms of composite cavity eigenmodes [9]. These modes, $u(z)$, are the solutions to Maxwell's equations for the entire CRVCL system. Neglecting transverse mode dependence, Maxwell's equations reduce to

$$
\frac{\mathrm{d}^{2}}{\mathrm{dz}^{2}} u(z)=-\mu_{o} \varepsilon(\mathrm{z}) \Omega^{2} \mathrm{u}(\mathrm{z})
$$

where $\Omega$ is the eigenfrequency, $\mu_{0}$ is the permeability constant, and $\varepsilon(z)$ describes the spatial variation in the permittivity due to different elements in the CRVCL structure. The solutions of (1) for the experimental situation of Fig. 3 (a) show the interaction between the resonators can be controlled by variations in the optical path lengths of the cavities. The detuning between the two cavities can be dynamically modified using current injection to depress the refractive index. For 
example, small changes in the optical path length of one resonator will modify its eigenfunction as shown in Fig. 5. Thus as the optical path of one cavity changes, the amplitude in that cavity will also change; if we extract light from that cavity, the laser output will be modulated. With the proper choice of coupling between the resonators, the sensitivity of the output on path length variations can be optimized. Moreover, the mode amplitude in the active cavity and its frequency can be designed to have negligible change, the latter enabling chirpless modulation of the laser.

The output intensity of the CRVCL device can also be modulated by reverse biasing the passive cavity. Fig. 3 (b) shows the output intensity as a function of active cavity current for several different reverse bias voltages. For a given active cavity current, the output intensity decreases with increasing reverse bias voltage. Since the reverse breakdown voltage of the passive cavity diode is about $-10.5 \mathrm{~V}$, considerable modulation occurs before an appreciable amount of current flows. The reverse bias modulation mechanism is therefore not related to carrier induced changes in the device but rather to the large field present in the active region. Thus, electroabsorption caused by the Franz-Keldysh effect is the most likely cause of the modulation under reverse bias conditions. Although the passive cavity is bulk GaAs, there will still be a small amount of absorption at the lasing wavelength $(990 \mathrm{~nm})$ induced by the applied voltage. This small amount of additional absorption inside the cavity can increase the loss sufficiently to drive the device below the lasing threshold. We also measured the shift in the lasing wavelength (see Fig. 4) under reverse bias operation of the passive cavity. With $1 \mathrm{~mA}$ of reverse bias current, the wavelength shifts from $998.7 \mathrm{~nm}$ (position A) to $998.9 \mathrm{~nm}$ (position C). The small shift of only $0.2 \mathrm{~nm}$ again indicates that heating does not play a significant role in the modulation under reverse bias operation.

In summary, we have discussed two different modulation mechanisms by which the emission from a coupled resonator vertical cavity laser can be varied for an active-passive structure. The first 
mechanism occurs under forward bias of the passive cavity and is due to a carrier depression of the index of refraction which changes the optical path length and hence the coupling between the two resonators. The modulation scheme agrees with our composite mode theory used to model the output of the coupled structure under forward bias operation. The second mechanism occurs under reverse bias of the passive cavity and is due to electroabsorption loss in the cavity. We are currently investigating other unique characteristics of the coupled cavity phenomena, such as frequency tuning, gain switching, and high speed modulation.

This research was performed at Sandia National Laboratories, a multiprogram laboratory operated by Sandia Corporation, for the United States Department of Energy under contract No. DE-AC04-94AL85000. 


\section{Figure Captions:}

Fig. 1: Schematic of a coupled resonator vertical cavity laser (CRVCL).

Fig. 2: Measured reflectance from a CRVCL with an 11.5 period middle DBR. The arrows denote the resonances. The inset shows calculated resonance splitting as a function of the number of middle DBR periods.

Fig. 3 : (a) Light output from the CRVCL for various forward bias passive cavity currents. (b) Light output from the CRVCL under reverse bias operation of the passive cavity.

Fig. 4 : Intensity as a function of active cavity current for passive cavity currents of $I_{p}=0 \mathrm{~mA}$, $I_{p}=-1 \mathrm{~mA}, I_{p}=20 \mathrm{~mA}$. The table shows the operating wavelength at positions $A$ through D.

Fig. 5 : Composite resonator eigenfunctions arising from a small reduction of the optical path length in one cavity. 


\section{References}

[1] W.T. Tsang, in Semiconductors and Semimetals; Vol. 22 B, edited by R.K.Willardson and A.C. Beer (Academic, New York, 1985), p.257.

[2] R.P. Stanley, R.Houdré, U.Oesterle, M. Ilegems, and C. Weisbuch, Appl. Phys. Lett. 65, 2093 (1994).

[3] P. Pellandini, R.P. Stanley, R. Houdré, U.Oesterle, M. Ilegems, and C. Weisbuch, Appl. Phys. Lett. 71, 864 (1997).

[4] P. Michler, M. Hilpert, and G. Reiner, Appl. Phys. Lett. 70, 2073 (1997).

[5] K.D. Choquette, W.W. Chow, H.Q. Hou, K.M. Geib, and B.E. Hammons, Proceeding of the SPIE- The International Society for Optical Engineering 3286, 134 (1998).

[6] J.F. Carlin, R.P. Stanley, P.Pellandini, U.Oesterle, and M.Ilegems, Proceeding of the SPIEThe International Society for Optical Engineering, 1999.

[7] J.A. Hudgings, R.J. Stone, S.F.Lim, W. Yuen, K.Y. Lau, and C.J. Chang-Hasnain, Appl. Phys. Lett. 73, 1796 (1998).

[8] K.D. Choquette, R. P. Schneider, Jr., K. L. Lear, and K. M. Geib, Electron. Lett. 30, 2043 (1994).

[9] W.W. Chow, IEEE J. Quantum Electron. QE-22, 1174 (1986); M. Rose, M. Lindberg, W. Chow, S. Koch, M. Sargent III, Phys. Rev. A 46, 603 (1992).

[10] G. Hasnain, K. Tai, L. Yang, Y.H. Yang, R.J. Fischer, J.D. Wynn, B. Weir, N.K. Dutta, and A.Y. Cho, IEEE J. Quantum Electron. QE-27, 1377 (1991). 
Figure 1

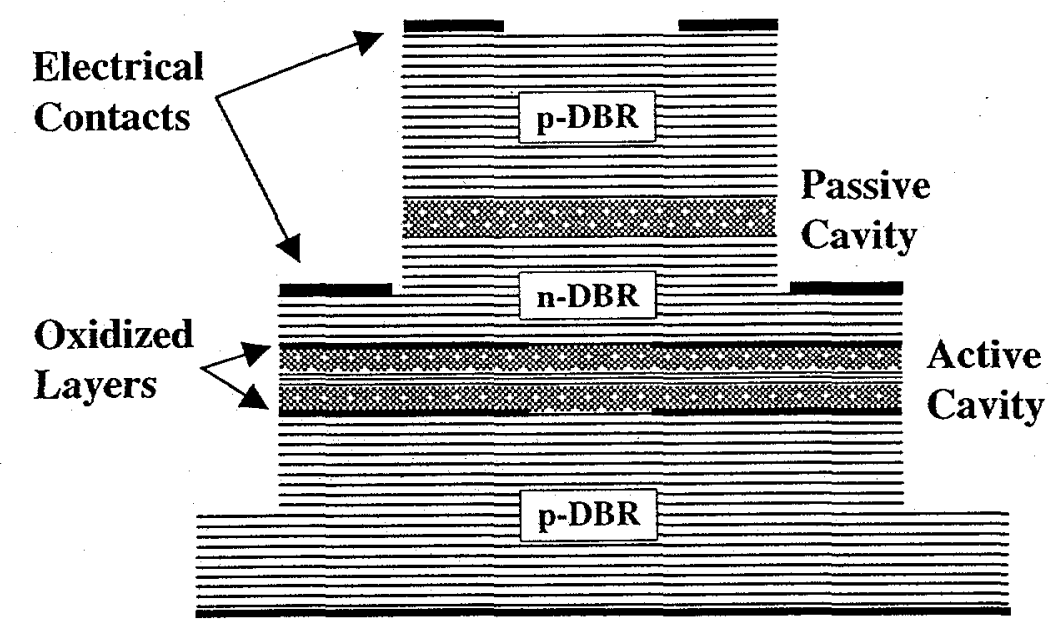


Figure 2:

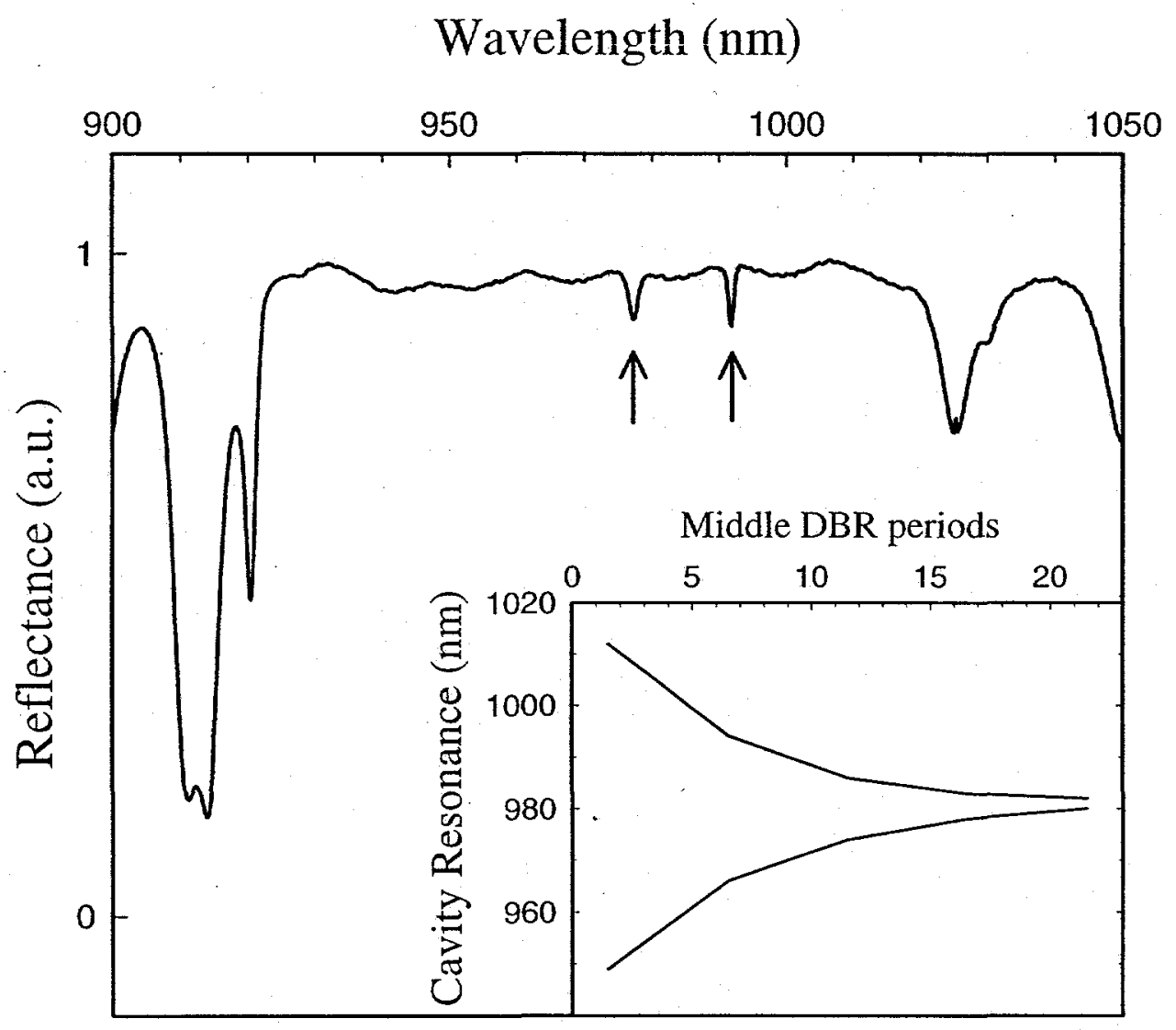


Figure 3:
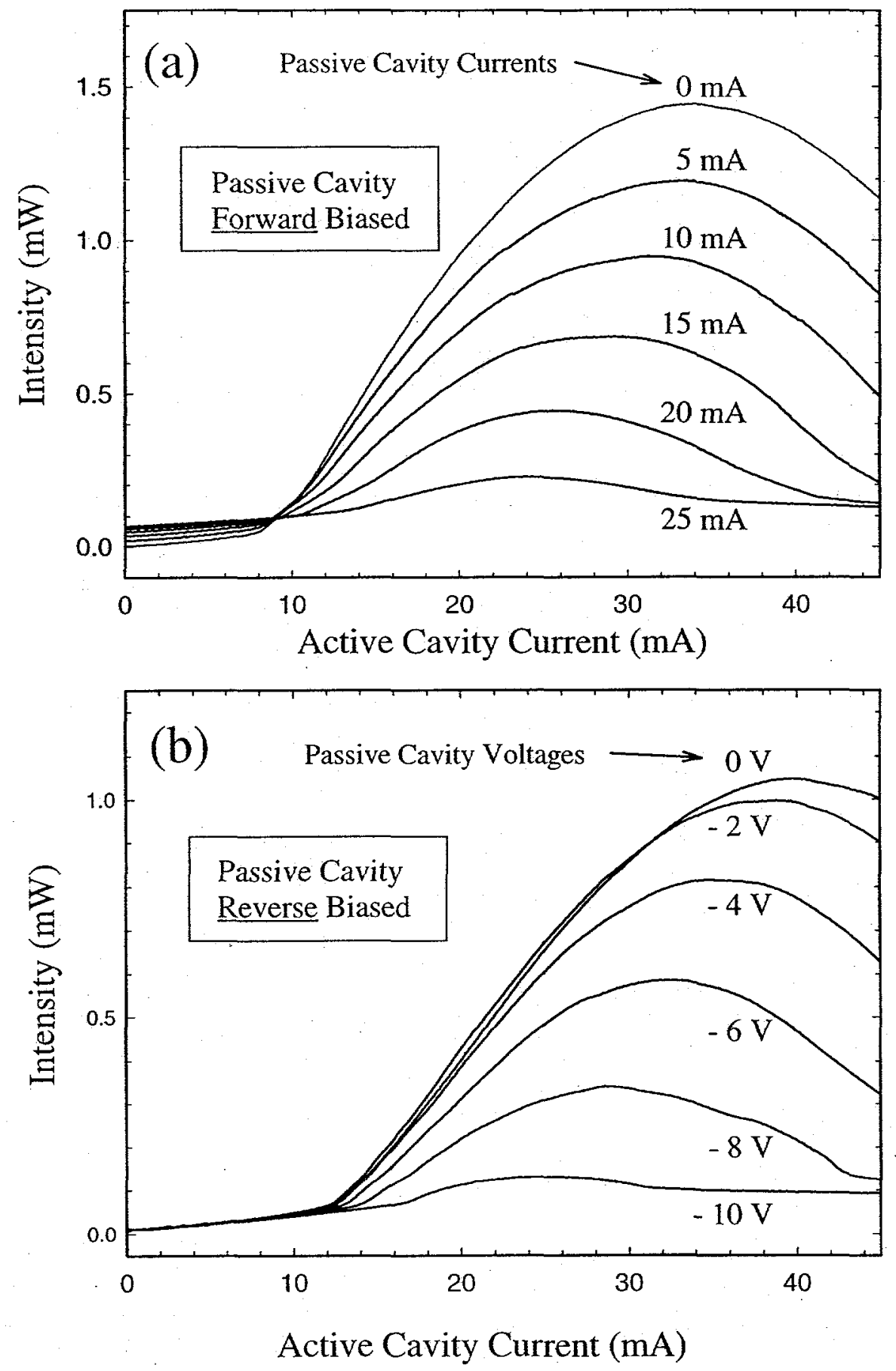
Figure 4:

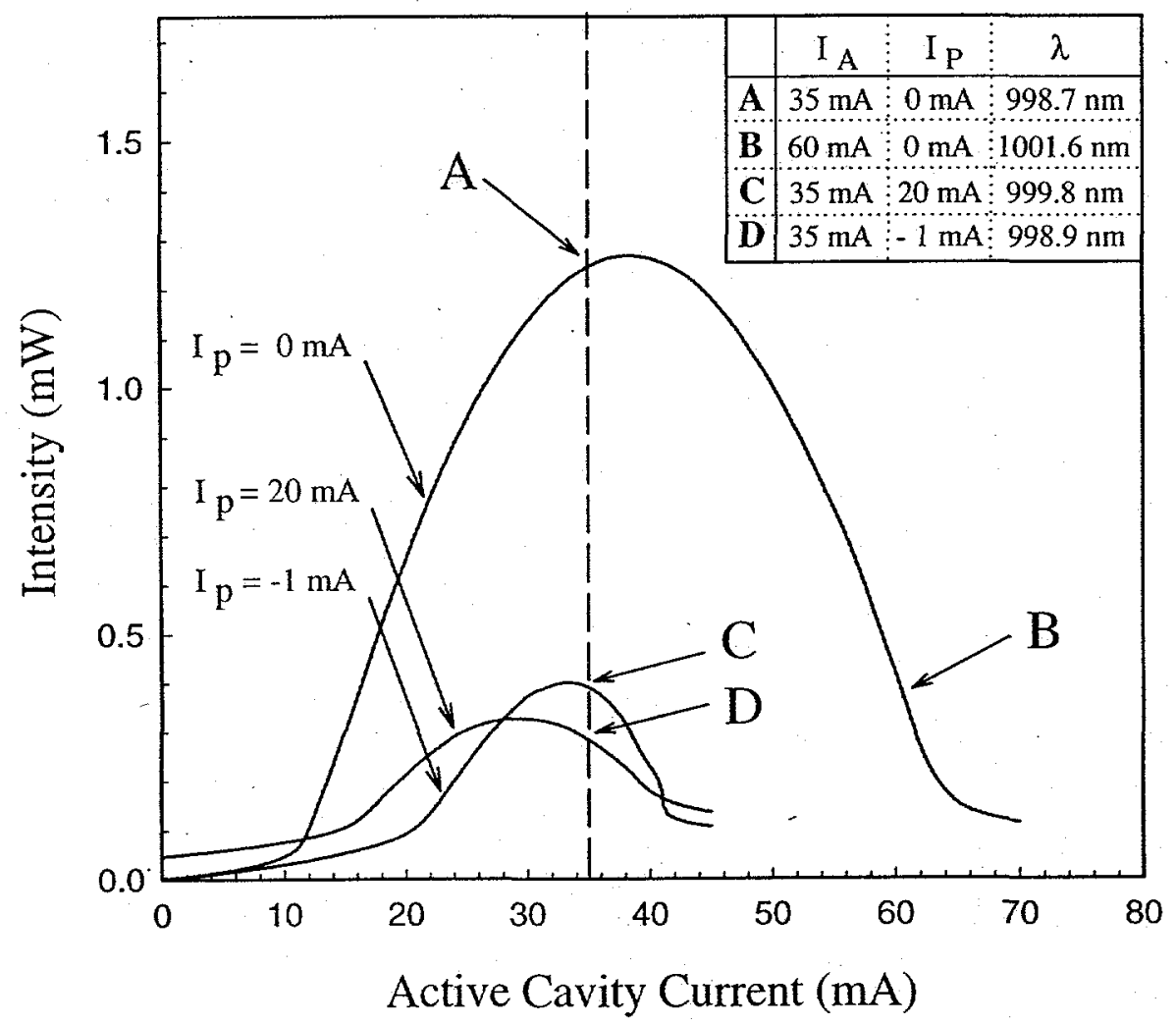


Figure 5:

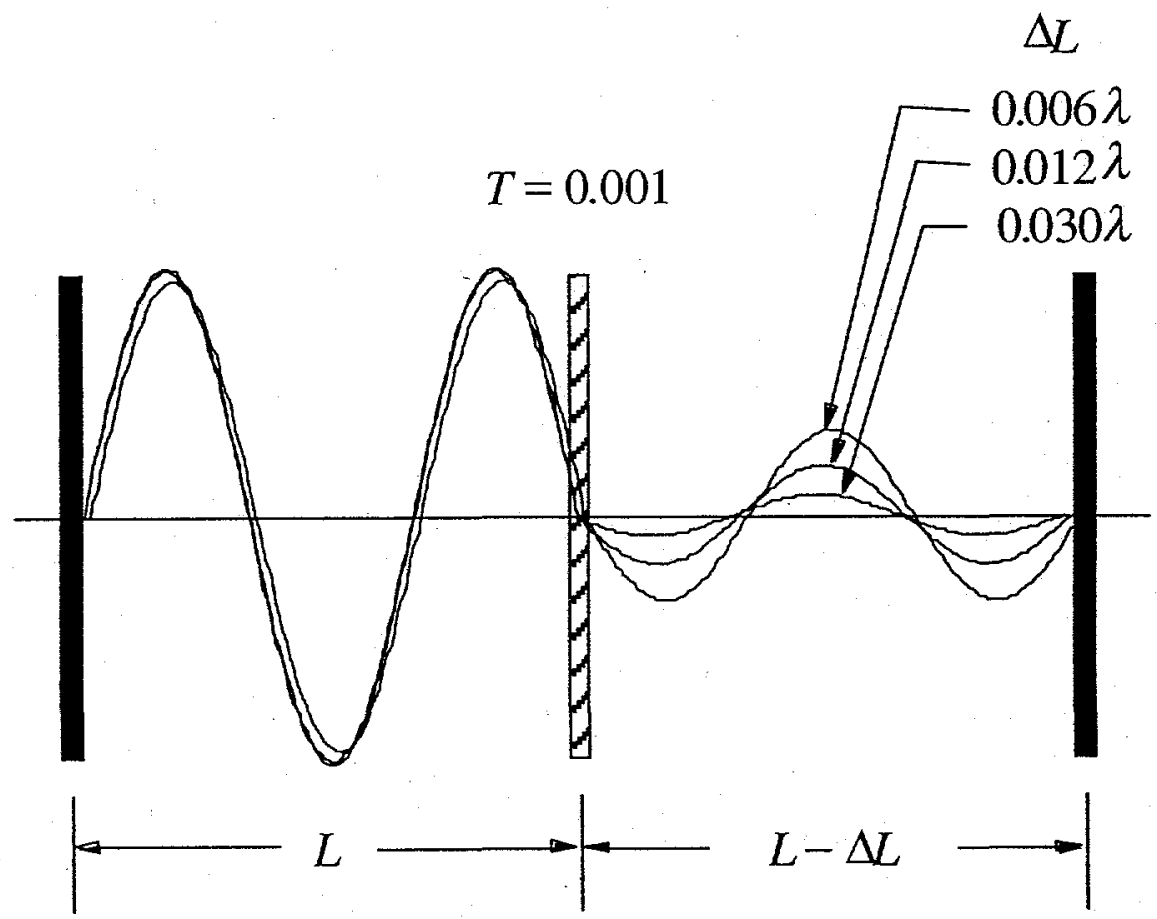

